\title{
Postpyloric decompression tube placement through a gastrostomy for malignant bowel obstruction
}

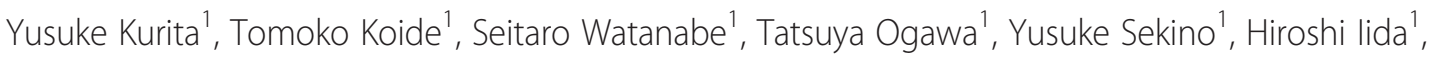

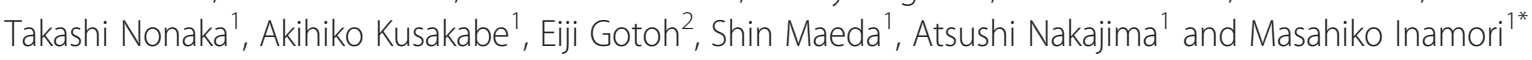

\begin{abstract}
Background: Malignant bowel obstruction affect a patient's quality of life, but, management of MBO is controversial. Case presentation: A 51-year-old woman who had been diagnosed as uterine cervix cancer 2 years ago and had undergone surgery, chemotherapy and radiotherapy, was admitted to our hospital. She was diagnosed as having a recurrence of peritoneal metastasis and bowel obstruction. For her nasal pain, we considered insertion of a postpyloric decompression tube through the gastrostomy instead of via the nasal cavity. After insertion of a percutaneous gastrostomy tube was performed endoscopically, we introduced a postpyloric decompression tube through her gastrostomy. She could be discharged home, and 91 days later, she died in her home under hospice care, as she had wished.
\end{abstract}

Conclusions: Insertion of a postpyloric decompression tube through a gastrostomy might be useful in the management of advanced cancer patients with bowel obstruction.

Keywords: Malignant bowel obstruction, Gastrostomy, Palliative care, Quality of life

\section{Background}

Malignant bowel obstruction (MBO), a common complication in patients with advanced cancer, can significantly affect a patient's quality of life [1-3]. However, management of MBO is controversial [4-6].

\section{Case presentation}

A 51-year-old woman who had been diagnosed as having stage $2 \mathrm{~b}$ uterine cervix cancer 2 years ago and had undergone surgery, chemotherapy and radiotherapy, was admitted to our hospital with nausea and abdominal pain. She was diagnosed as having a recurrence of peritoneal metastasis with complicating ascites and bowel obstruction. We first treated her conservatively however, a month later, her symptoms recurred and a postpyloric decompression tube was introduced via the nasal cavity. After the procedure,

\footnotetext{
* Correspondence: inamorim@med.yokohama-cu.ac.jp

'Gastroenterology Division, Yokohama City University Hospital, 3-9 Fukuura, Kanazawa-ku, Yokohama 236-0004, Japan

Full list of author information is available at the end of the article
}

she complained of severe nasal pain and expressed her wish for treatment by a different method.

We therefore considered insertion of a postpyloric decompression tube through the gastrostomy instead of via the nasal cavity. Following obtainment of informed consent, insertion of a percutaneous gastrostomy tube was performed endoscopically (Figure 1). Two weeks later, we introduced a postpyloric decompression tube through her gastrostomy instead of via the nasal cavity. The postpyloric decompression was effective (Figure 2), she was discharged home, and 91 days later, she died in her home under hospice care, as she had wished.

\section{Conclusions}

Palliation of symptoms is the treatment goal terminal disease patients with MBO. Hospitalization and conservative management by nasogastric tube decompression and bowel rest is the first step in the management of MBO. However, when continuous postpyloric decompression is required, insertion of the postpyloric decompression tube through the gastrostomy instead of via the nasal cavity

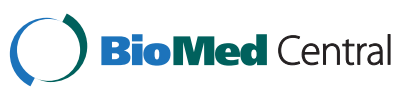




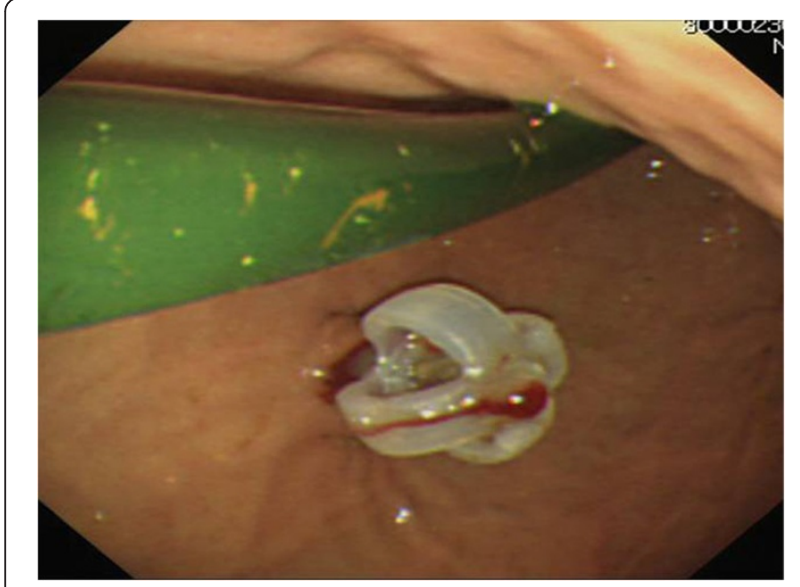

Figure 1 Percutaneous gastrostomy tube insertion was performed endoscopically, while a transnasal postpyloric decompression tube was present in her stomach.

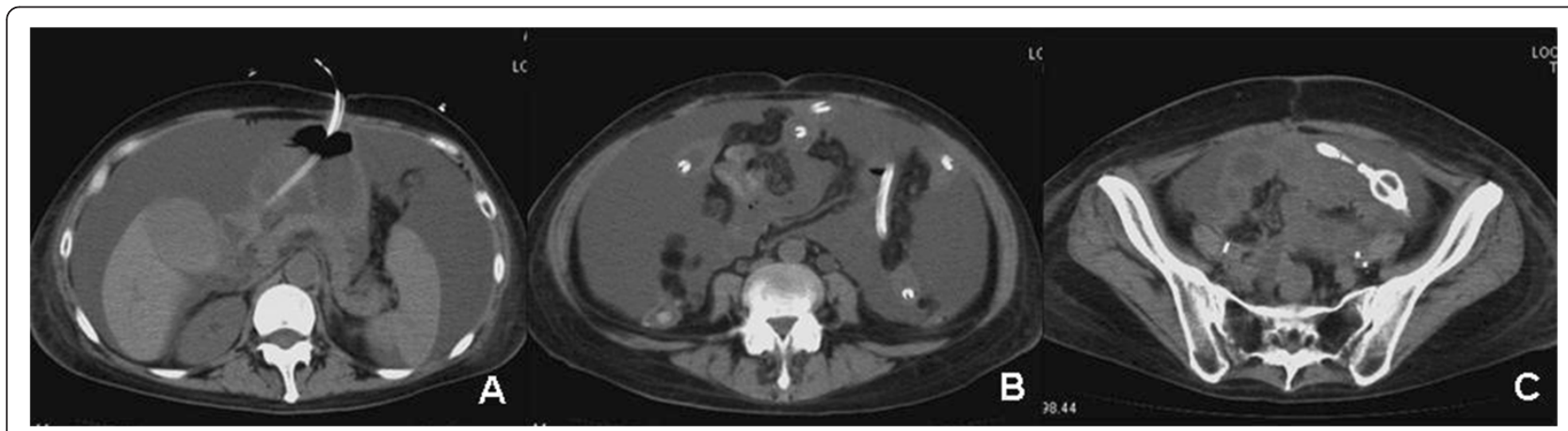

Figure 2 Postpyloric decompression tube was inserted through the gastrostomy.

may be a reasonable approach, especially in patients hesitating to undergo tube placement via the conventional nasal approach and/or in gynecologic malignancies patients who are not good surgical candidates.

In conclusion, insertion of a postpyloric decompression tube through a gastrostomy might be useful in the management of advanced cancer patients with bowel obstruction.

\section{Consent}

Written informed consent was obtained from the patient and her family for publication of this Case report and any accompanying images. A copy of the written consent is available for review by the Editor of this journal.

\section{Competing interests}

The authors declare that they have no competing interest.

\section{Authors' contributions}

TK, AK and Ml gave information about gastrostomy. SW and MI collect clinical data. YS, HI and TN gave information about endoscopy. AK gave information about palliative care. YK, TO and MI wrote the manuscript. EG, SM and AN supervised the manuscript. All authors read and approved the final manuscript.

\section{Acknowledgements}

Special thanks to medical staff of Yokohama City University Hospital.

\section{Author details}

${ }^{1}$ Gastroenterology Division, Yokohama City University Hospital, 3-9 Fukuura, Kanazawa-ku, Yokohama 236-0004, Japan. ²Department of Medical Education, Yokohama City University School of Medicine, Yokohama, Japan.

Received: 21 December 2012 Accepted: 23 May 2013

Published: 3 June 2013

\section{References}

1. Mori M, Bruera E, Dev R: Complications of a gastrostomy tube used for decompression of an inoperable bowel obstruction in a patient with advanced cancer. J Pain Symptom Manage 2009, 38:466-472.

2. Mangili G, Aletti G, Frigerio L, Franchi M, Panacci N, Viganò R, DE Marzi P Zanetto F, Ferrari A: Palliative care for intestinal obstruction in recurrent ovarian cancer: a multivariate analysis. Int J Gynecol Cancer 2005, 15:830-835.

3. Meyer $L$, Pothuri B: Decompressive percutaneous gastrostomy tube use in gynecologic malignancies. Curr Treat Options Oncol 2006, 7:111-120.

4. Dolan EA: Malignant bowel obstruction: a review of current treatment strategies. Am J Hosp Palliat Care 2011, 28:576-582.

5. Rezk Y, Timmins PF 3rd, Smith HS: Review article: palliative care in gynecologic oncology. Am J Hosp Palliat Care 2011, 28:356-374.

6. Roeland E, von Gunten CF: Current concepts in malignant bowel obstruction management. Curr Oncol Rep 2009, 11:298-303.

doi:10.1186/1756-0500-6-217

Cite this article as: Kurita et al: Postpyloric decompression tube placement through a gastrostomy for malignant bowel obstruction. BMC Research Notes 2013 6:217. 